\title{
LncRNA TMEM220-AS1 suppresses hepatocellular carcinoma cell proliferation and invasion by regulating the TMEM220/ $\beta$-catenin axis
}

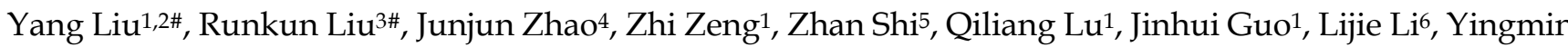 \\ $\mathrm{Yao}^{3}, \mathrm{Xin} \mathrm{Liu}^{2}$ and Qiuran $\mathrm{Xu}^{2 \bowtie}$ \\ 1. The Medical College of Qingdao University, Qingdao, 266071, China. \\ 2. The Key Laboratory of Tumor Molecular Diagnosis and Individualized Medicine of Zhejiang Province, Zhejiang Provincial People's Hospital, Affiliated People's \\ Hospital, Hangzhou Medical College, Hangzhou 310014, China. \\ Department of Hepatobiliary Surgery, The First Affiliated Hospital of Xi'an Jiaotong University, Xi'an 710061, China. \\ Graduate Department, Bengbu Medical College, Bengbu 233030, China. \\ The Second Clinical Medical College, Zhejiang Chinese Medical University, Hangzhou 310053, China. \\ Department of Obstetrics and Gynaecology, Affiliated Zhejiang Hospital, Zhejiang University School of Medicine, Hangzhou 310013, China. \\ \#These authors contributed equally to this work.
}

$\triangle$ Corresponding authors: Prof. Xin Liu, E-mail: liuxindongdong@sina.com; Prof. Qiuran Xu, E-mail: liuxin@hmc.edu.cn.

(C) The author(s). This is an open access article distributed under the terms of the Creative Commons Attribution License (https://creativecommons.org/licenses/by/4.0/). See http:/ /ivyspring.com/terms for full terms and conditions.

Received: 2021.05.31; Accepted: 2021.09.14; Published: 2021.09.24

\begin{abstract}
Long non-coding RNAs (IncRNAs) are critical drivers and suppressors of human hepatocellular carcinoma (HCC). The downregulation of transmembrane protein 220 antisense RNA 1 (TMEM220-AS1) is correlated with poor prognosis in HCC. Nevertheless, the role of TMEM220-AS1 in HCC and the underlying mechanism remains unclear. In this study, TMEM220-AS1 levels were markedly reduced in HCC tissues compared with noncancerous tissues. TMEM220-AS1 downregulation was confirmed in HCC cell lines. TMEM220-AS1 expression was associated with tumor stage, venous infiltration, tumor size, and survival of HCC patients. TMEM220-AS1 overexpression suppressed the migration, invasion, and proliferation of HCC cells. Interestingly, ectopic expression of TMEM220-AS1 increased TMEM220 levels in HCC cells. Decreased TMEM220 levels were observed in HCC tissues and cell lines. TMEM220 expression was positively correlated with TMEM220-AS1 levels in HCC tissue samples and TMEM220 downregulation was significantly correlated with reduced patient survival. TMEM220 overexpression suppressed HCC cell proliferation and mobility. TMEM220 knockdown eliminated the suppressive effect of TMEM220-AS1 in HCCLM3 cells. Mechanistically, TMEM 220 overexpression reduced the nuclear accumulation of $\beta$-catenin and decreased MYC, Cyclin D1, and Snaill mRNA levels in HCCLM3 cells. BIO, a GSK3 $\beta$ inhibitor, eliminated TMEM 220 -induced Wnt/ $\beta$-catenin pathway inactivation and inhibited HCC cell proliferation and mobility. In conclusion, TMEM220-AS1 and TMEM220 were expressed at low levels in HCC patients. TMEM220-AS1 inhibited the malignant behavior of $\mathrm{HCC}$ cells by enhancing TMEM220 expression and subsequently inactivating the $\mathrm{Wnt} / \mathrm{\beta}$-catenin pathway.
\end{abstract}

Key words: Hepatocellular carcinoma; TMEM220-AS1; TMEM220; $\beta$-catenin; Tumor progression

\section{Introduction}

The most recent data from the International Agency for Research on Cancer (IARC) indicates that primary liver cancer is the fifth most common cancer and the second leading cause of cancer-related deaths in China [1]. Hepatocellular carcinoma (HCC) accounts for approximately $90 \%$ of all liver cancers. The five-year survival rate for patients with HCC who undergo hepatectomy is less than $20 \%$ in China [2]. Thus, multiple studies have focused on investigating the mechanisms underlying hepatocarcinogenesis and developing new drugs targeting HCC.

RNA transcripts longer than 200 nucleotides which lack significant protein-coding ability are called long noncoding RNAs (lncRNAs) [3]. An increasing number of studies have demonstrated the critical functions of lncRNAs in the occurrence and progression of human HCC [4-6]. We have previously investigated the impact of lncRNAs, including 
MCM3AP-AS1, PICSAR, LINC01123, and A1BG-AS1, in HCC tumor progression [7-10]. MCM3AP-AS1 is highly expressed in HCC, and its overexpression predicts poor prognosis in HCC [7]. MCM3AP-AS1 competitively binds miR-194-5p to enhance FOXA1 expression and facilitates HCC cell growth [7]. LINC01123 is a tumor-promoting factor that exerts an oncogenic effect in HCC cell proliferation, migration, and invasiveness by modulating the miR-34a-5p/ TUFT1 axis [9]. The upregulation of PICSAR and its association with poor prognosis were reported in our previous study [8]. PICSAR contributes to HCC progression by modulating the miR-588/EIF6 axis and activating the PI3K/AKT/mTOR pathway [8]. A1BG-AS1 functions as a tumor suppressor and inhibits the proliferation, migration, and invasion of tumor cells by targeting miR-216-5p in HCC [10]. A recent study reported the downregulation of transmembrane protein 220 antisense RNA 1 (TMEM220-AS1) and its copy number variation in HCC [11]. Moreover, low TMEM220-AS1 expression predicts poor prognosis in HCC [11]. Nevertheless, the role of TMEM220-AS1 in HCC and the underlying mechanism of action remains unclear. TMEM220-AS1 is an antisense lncRNA of TMEM220. High TMEM220 methylation and its downregulation have been detected in gastric cancer tissues [12]. However, it is unknown whether TMEM220 acts as a functional target of TMEM220-AS1.

Here, we analyzed TMEM220-AS1 and TMEM220 expression in HCC and adjacent nontumor tissues and determined their prognostic significance. We investigated the effects of TMEM220AS1 and TMEM220 on cell proliferation, invasion, and migration. The potential pathways regulated by TMEM220 were investigated using Kyoto Encyclopedia of Genes and Genomes (KEGG) enrichment analysis. Our data showed that the levels of TMEM220-AS1 and TMEM220 were reduced in HCC specimens and were associated with poor prognosis. TMEM220-AS1 suppressed the malignant behavior of tumor cells by targeting the TMEM220/ $\beta$-catenin axis in HCC.

\section{Material and methods}

\section{HCC patients and specimens}

Eighty pairs of tumors and adjacent non-tumor tissues were collected from HCC patients who underwent $\mathrm{R} 0$ resection at the First Affiliated Hospital of Xi'an Jiaotong University between January 2012 and March 2014. Patients who were pathologically confirmed to have HCC and had complete clinical data were enrolled after providing written informed consent. Patients with HCC who had received preoperative treatment or had other tumors were excluded. The specimens were maintained at $-80{ }^{\circ} \mathrm{C}$. The study protocol (No: XJTU1AF2020LSK-123) was approved by the Ethics Committee of the First Affiliated Hospital of Xi'an Jiaotong University. Table 1 presents the clinical characteristics of the HCC patients.

Table 1. Correlation between TMEM220-ASI expression and clinicopathologic characteristics in hepatocellular carcinoma

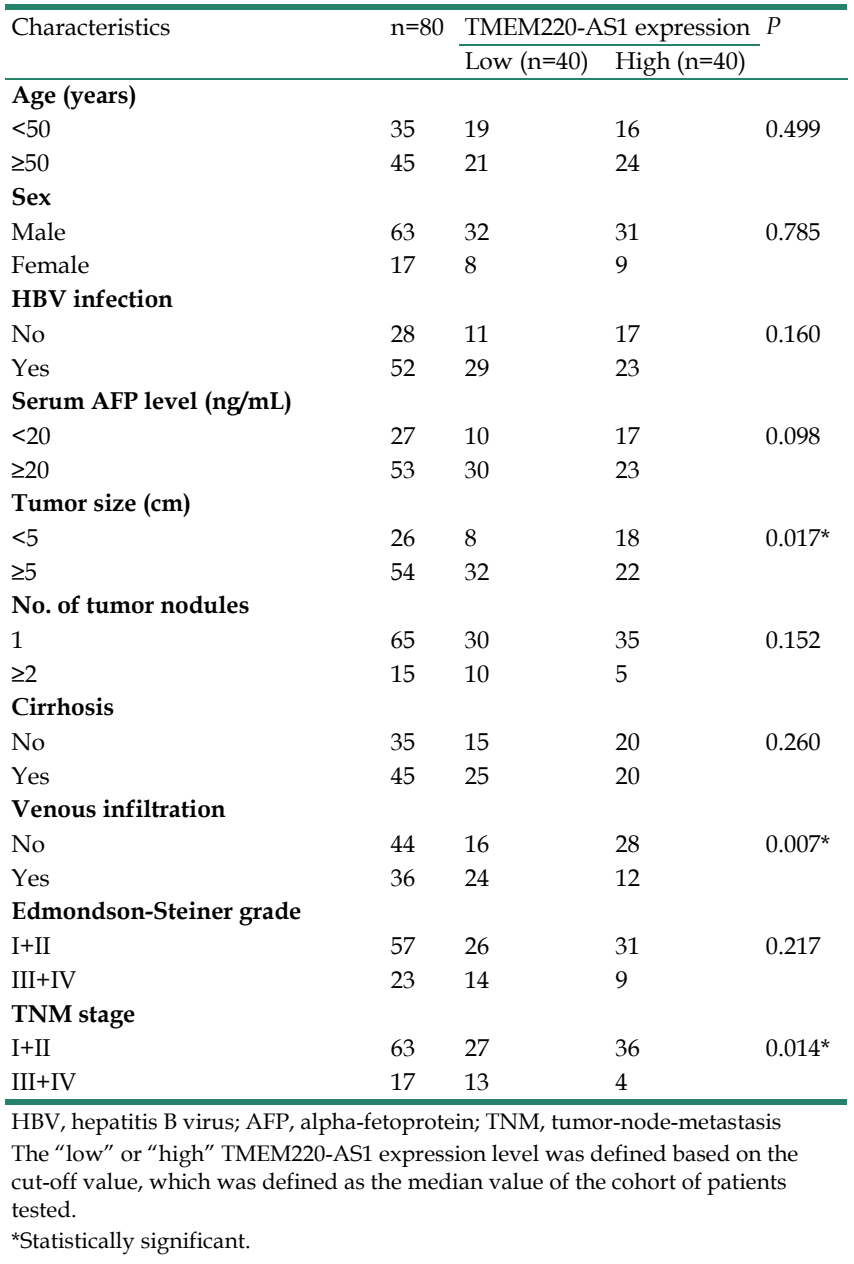

\section{Cell culture and transfection}

Human HCC cell lines (HCCLM3, Hep3B, HepG2, MHCC97H, and Huh7) were preserved in our lab [13]. The normal hepatic cell line, MIHA, was obtained from Bnbio (Beijing, China) and cultured under standard conditions. TMEM220-AS1 and TMEM220 expression plasmids were generated by inserting cDNA into the pcDNA3.1 vector (V79020, Invitrogen, Carlsbad, CA, USA). TMEM220 shRNA (shTMEM220) was provided by GenePharma (Shanghai, China). The vectors were transfected into HCC cells using the Qiagen Effectene transfection reagent (301427, Valencia, CA, USA). 


\section{RT-qPCR}

RNA was extracted using TRIzol reagent (15596018, Invitrogen). TIANScript RT Kit (KR104, Tiangen Biotech, Beijing, China) was used for reverse transcription. Amplification was performed using the Bio-Rad CFX96 Touch Real-Time PCR Detection System (Bio-Rad, Hercules, CA, USA) and FS Universal SYBR Green Master kit (4913850001, Roche, Shanghai, China) following the manufacturer's instructions. The $2^{-\Delta \Delta \mathrm{CT}}$ method was used to analyze relative changes in gene expression. The primers used are listed in Supplementary Table 1.

\section{Western blotting (WB) analysis}

Protein isolation was carried out using the RIPA Lysis Buffer System (sc-24948, Santa Cruz, CA, USA) and the concentration was measured using the Bradford protein assay kit (P0006, Beyotime, Shanghai, China). Then, $20 \mu \mathrm{g}$ protein was subjected to SDS-PAGE $(10 \%)$ and the bands transferred to PVDF membranes (IPVH00010, Millipore, Bedford, MA, USA). The membranes were incubated overnight at $4{ }^{\circ} \mathrm{C}$ with anti-TMEM220 (PA5-69312, Thermo Fisher Scientific, Waltham, MA, USA), anti- $\beta$-catenin (\#8480, Cell Signaling Technology, Beverly, MA, USA), anti- $\beta$-actin (66009-1-Ig, Proteintech, Wuhan, China), and histone H3 (PTM-1001RM, PTM BIO, Hangzhou, China). The membranes were then incubated with HRP-bound secondary antibody (A0208 and A0216, Beyotime) and detected using an ECL system (WBLUF0500, Millipore). The resulting bands were scanned using an Amersham Imager 680 (GE Healthcare Life Sciences, Pittsburgh, PA, USA).

\section{Cell proliferation assay}

For the cell counting kit-8 (CCK-8) assay, transfected HCC cells were suspended in complete DMEM and seeded into 96-well plates $\left(2 \times 10^{3}\right.$ per well). The plates were supplemented with $10 \mu \mathrm{L}$ CCK-8 solution (CK04, Dojindo Laboratories, Dojindo, Japan) per well and incubated for $3 \mathrm{~h}$ at 37 ${ }^{\circ} \mathrm{C}$. The absorbance value (OD) was measured at 450 nm using a Multiskan FC microplate reader (Thermo Fisher Scientific). The EdU assay was conducted using the Cell-Light ${ }^{\mathrm{TM}}$ EdU Apollo®488 In Vitro Imaging Kit (C10310-1, RIBOBIO, Guangzhou, China) following the manufacturer's protocol, as previously described [7].

\section{Transwell assay}

The migratory and invasive abilities of HCC cells were assessed using transwell chambers with or without Matrigel (356234, BD Biosciences, Franklin Lakes, NJ, USA). Cells $\left(3 \times 10^{4}\right)$ in serum-free DMEM medium were seeded on the upper chamber and DMEM containing $10 \%$ FBS was added as a chemoattractant to the lower chamber. After $24 \mathrm{~h}$ incubation, $0.1 \%$ crystal violet was used to visualize the migrated or invaded HCC cells. Images of the membrane area were captured randomly and the cells counted.

\section{TCGA data analysis}

TCGA data analysis was performed using the Gene Expression Profiling Interactive Analysis (GEPIA) webserver (http://gepia.cancer-pku.cn/) as previously described [14].

\section{Statistical analysis}

Analysis of variance (ANOVA) with Tukey's multiple comparison test, Student's $t$-test, chi-squared test, and Mann-Whitney U-test were performed using GraphPad Prism version 8 (GraphPad Inc., San Diego, CA, USA). Survival in the two HCC subgroups was compared using the Kaplan-Meier method and log-rank test. Results from at least three independent repeats are shown as mean \pm S.D. Statistical significance was set at $P<0.05$.

\section{Results}

\section{The downregulation of TMEM220-AS1 is correlated with HCC patients' survival}

First, we demonstrated that TMEM220-AS1 expression was significantly reduced in tumor tissue samples compared with adjacent noncancerous tissues ( $P=0.001$, Figure $1 \mathrm{~A})$. Consistent with our data, TCGA data also indicated that TMEM220-AS1 expression in HCC samples was markedly lower than that in normal tissues $(P<0.0001$, Supplementary Figure 1A). The expression of TMEM220-AS1 was downregulated in HCC cell lines, including HCCLM3, Huh7, Hep3B, HepG2, and MHCC97H, compared with MIHA cells $(P<0.05$, Figure $1 \mathrm{~B})$. The data in Table 1 show that low levels of TMEM220-AS1 were associated with malignant clinical parameters of HCC, including advanced TNM stage $(P=0.014)$, venous infiltration $(P=0.007)$, and tumor size $>5 \mathrm{~cm}$ $(P=0.017)$. A close correlation between TMEM220-AS1 expression and the pathological stage of HCC was identified by analyzing the TCGA data $(P<0.0001$, Supplementary Figure 1B). Our follow-up results and TCGA data consistently suggested that the reduced TMEM220-AS1 levels correlated with poor prognosis in HCC patients $(P<0.05$, Figure $1 C$ and Supplementary Figure 1C). Thus, our data indicates that TMEM220-AS1 is a potential predictive marker for HCC prognosis. 

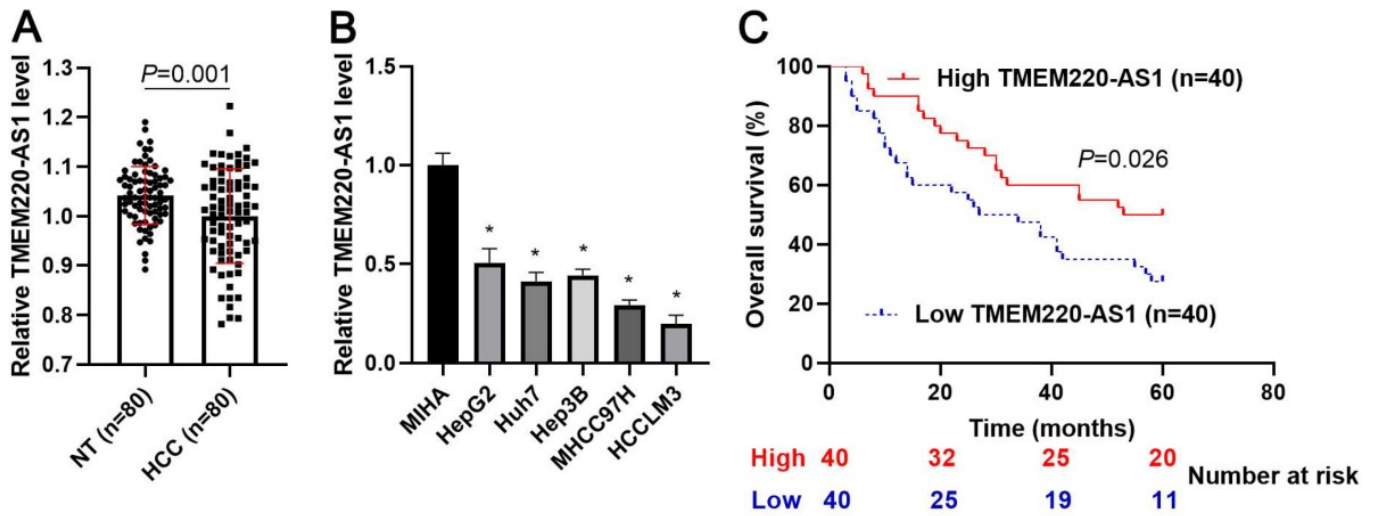

Figure 1. Expression and clinical significance of TMEM220-AS1 in HCC. (A) TMEM220-AS1 levels were detected in 80 pairs of HCC and nontumor (NT) tissues. (B) TMEM220-AS1 expression was assessed in MIHA, HCCLM3, Huh7, HepG2, Hep3B, and MHCC97H cells. (C) The survival of HCC patients with low versus high TMEM220-AS1 expression was compared. ${ }^{*} P<0.05$.
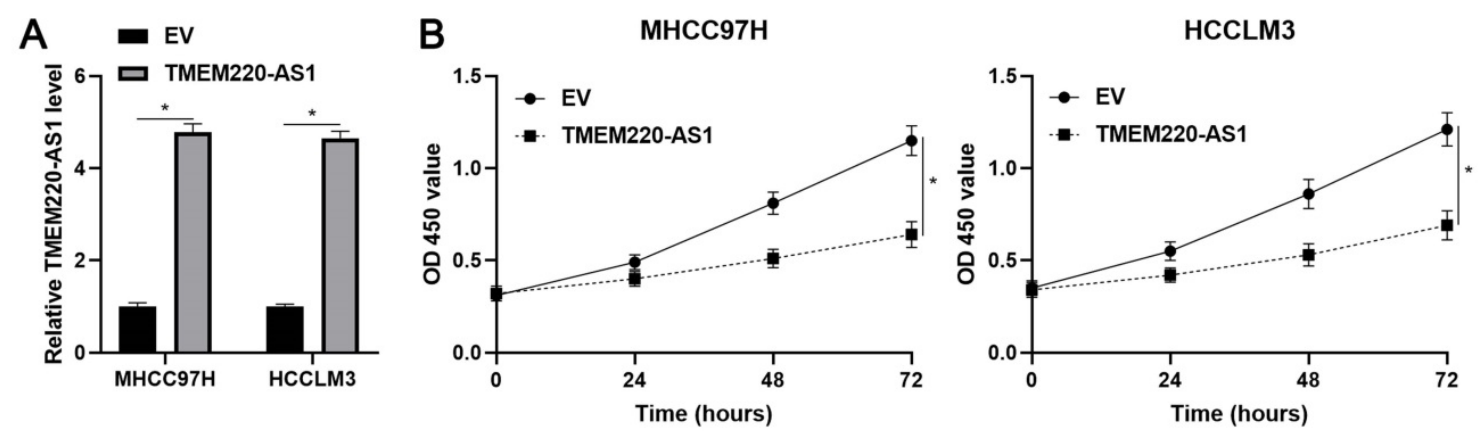

C
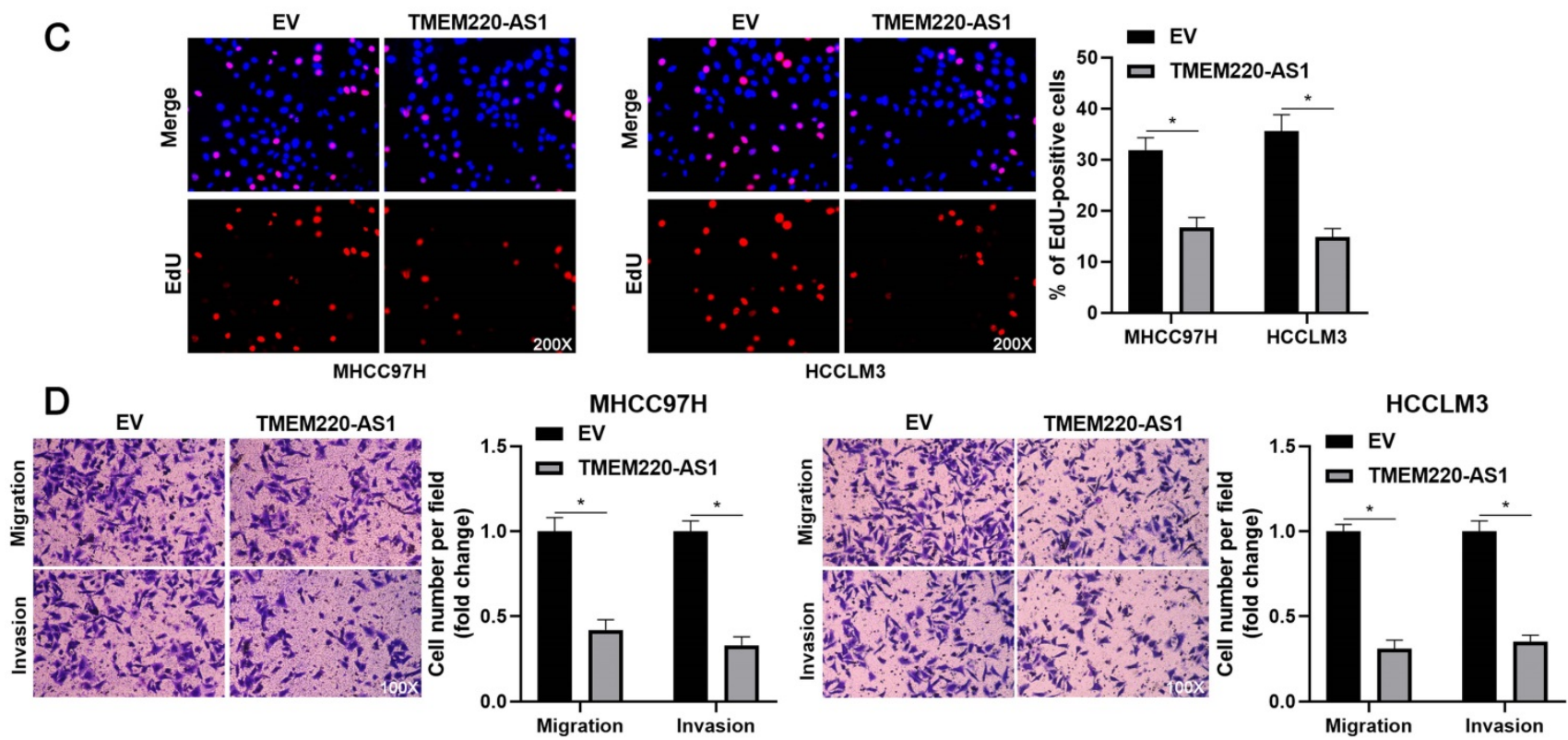

Figure 2. TMEM220-AS1 inhibits HCC cell proliferation and mobility. (A) MHCC97H and HCCLM3 cells were transfected with pCDNA3.1 vector carrying TMEM220-AS1 or with empty vector (EV) and then analyzed for TMEM220-AS1 expression using RT-qPCR. (B) TMEM220-AS1 overexpression suppressed HCC cell viability, as shown using CCK-8 assay. (C) EdU assay confirmed that TMEM220-AS1 overexpression decreased HCC cell proliferation. (D) The number of migrated and invaded HCC cells was reduced by TMEM220-AS1 overexpression. $* P<0.05$.

\section{TMEM220-AS1 suppresses the biological behavior of HCC cells}

To determine the effects of TMEM220-AS1 on the malignant behavior of HCC cells, TMEM220-AS1 was ectopically expressed in MHCC97H and HCCLM3 cells $(P<0.05$, Figure $2 \mathrm{~A})$. The viability of HCC cells was significantly suppressed by TMEM220-AS1 overexpression $(P<0.05$, Figure $2 \mathrm{~B})$, as indicated by the CCK-8 assay. The cell proliferation assay confirmed that TMEM220-AS1 overexpression markedly reduced the percentage of EdU-positive HCC cells $(P<0.05$, Figure $2 \mathrm{C})$. Subsequent transwell assays showed that TMEM220-AS1 overexpression 
reduced the number of migrated and invaded HCC cells $(P<0.05$, Figure 2D). Therefore, these results suggest that TMEM220-AS1 suppresses the proliferation and invasiveness of HCC cells.

\section{TMEM220 is upregulated by TMEM220-AS1 and downregulated in HCC}

TMEM220-AS1 is an antisense lncRNA of TMEM220. Thus, we elucidate the regulatory effect of TMEM220-AS1 on TMEM220 expression. As expected, we found that TMEM220-AS1 overexpression significantly increased TMEM220 mRNA and protein expression in MHCC97H and HCCLM3 cells $(P<0.05$, Figure $3 \mathrm{~A}$ and $3 \mathrm{~B})$. However, the overexpression of TMEM220-AS1 did not affect the stability of TMEM220 mRNA in HCC cells (Supplementary Figure 2). On this basis, our clinical and TCGA data demonstrated that TMEM220 mRNA expression in HCC was significantly lower than in adjacent non-tumor tissues $(P<0.0001$, Figure $3 C$ and Supplementary Figure 3A). TMEM220 levels were lower in HCC cell lines compared with MIHA cells $(P<0.05$, Figure 3D). Moreover, positive correlation between TMEM220 mRNA and TMEM220-AS1 expression was confirmed in HCC tissue samples $(P<0.0001$, Figure 3E and Supplementary Figure 3B). HCC patients with low TMEM220 expression had significantly shorter overall survival than patients with high TMEM220 expression ( $P=0.0028$, Figure $3 F$ ). TCGA data also confirmed that low TMEM220 mRNA expression was associated with advanced pathological stage and poor HCC prognosis $(P<0.01$, Supplementary Figure $3 \mathrm{C}$ and 3D). Collectively, TMEM220 was a target for TMEM220-AS1, and its low expression was associated with poor clinical outcome in HCC.
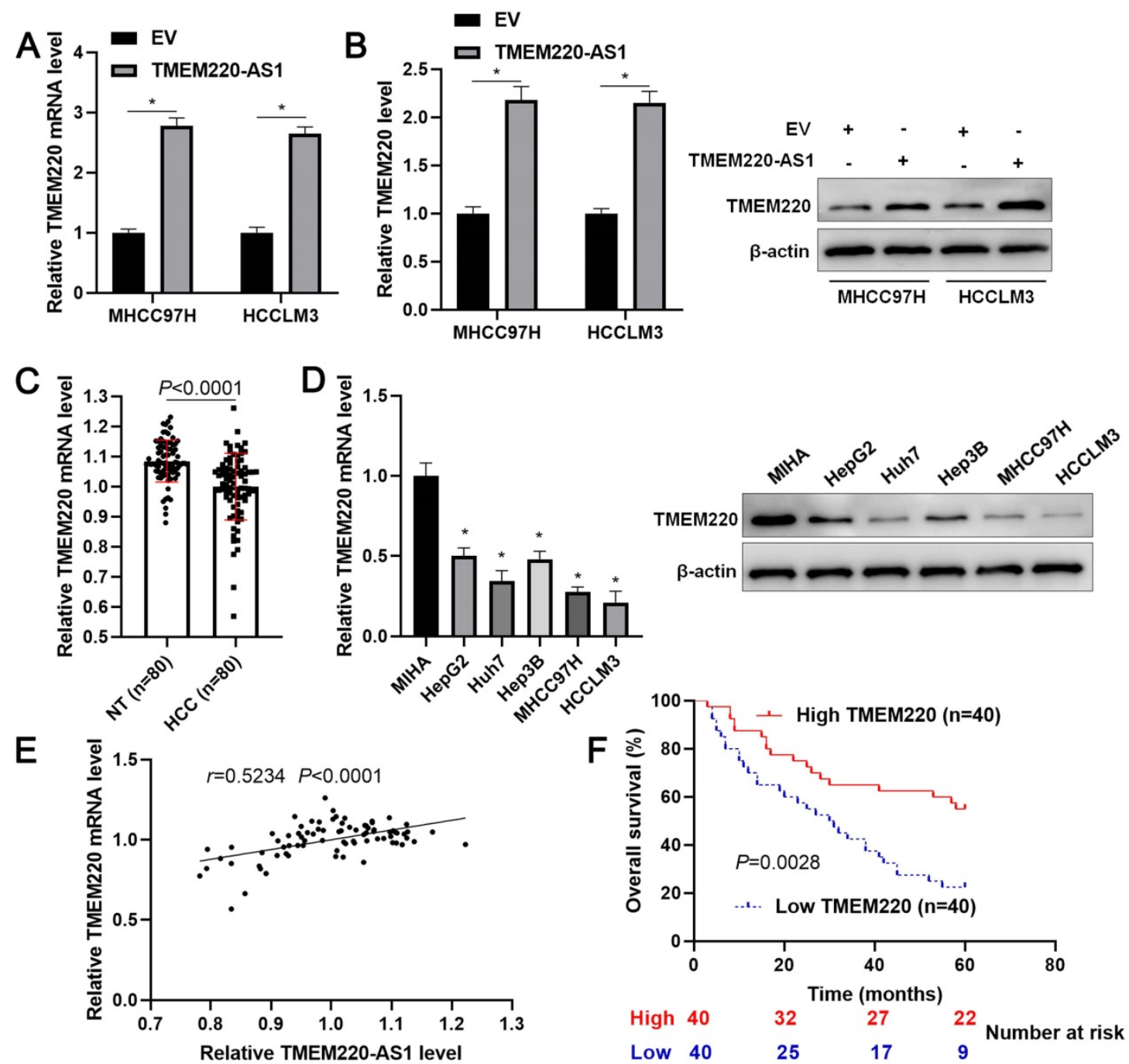

Figure 3. TMEM220 is a target of TMEM220-AS1. (A) HCC cells were transfected with pcDNA3.1 vector carrying TMEM220-AS1 or with empty vector (EV) and TMEM220 mRNA was detected using RT-qPCR. (B) Western blotting analysis revealed that TMEM-220-AS1 overexpression upregulated TMEM220 protein levels in HCC cells. (C) The levels of TMEM220 mRNA were measured in 80 pairs of HCC and nontumor (NT) tissues. (D) TMEM220-AS1 expression was assessed in MIHA, HCCLM3, Huh7, HepG2, Hep3B, and MHCC97H cells. (E) TMEM220-AS1 was positively associated with TMEM220 mRNA expression in HCC tissue samples. (F) The survival of HCC patients with low versus high TMEM220 expression was compared. $* P<0.05$. 


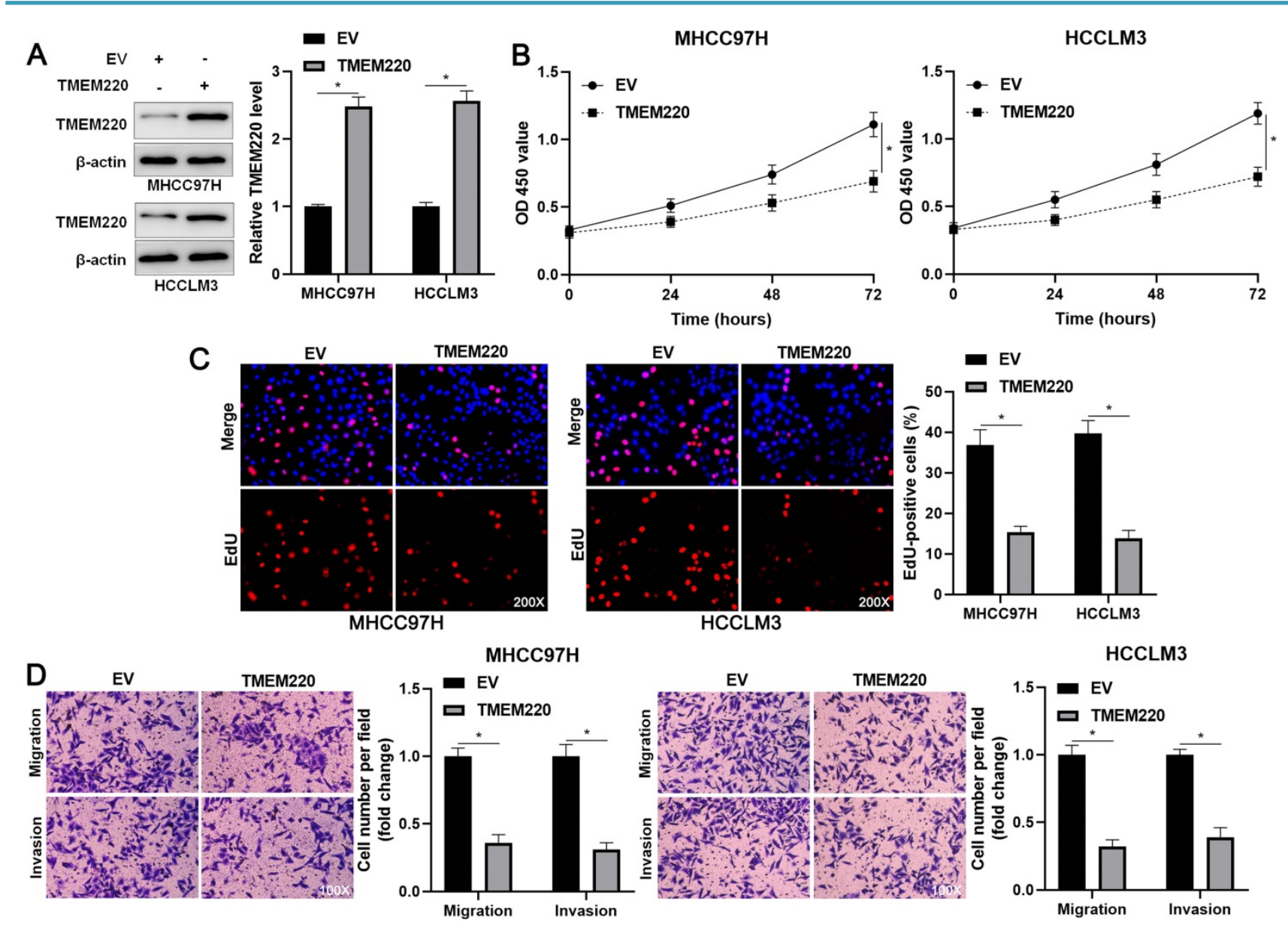

Figure 4. TMEM220 suppresses the migration, invasion, and proliferation of HCC cells. (A) MHCC97H and HCCLM3 cells were transfected with pcDNA3.1 vector carrying TMEM 220 or with empty vector (EV) and TMEM220 analyzed using western blotting analysis. (B) TMEM220 overexpression suppressed HCC cell viability, as determined using CCK-8 assay. (C) EdU assay confirmed that TMEM220 overexpression weakened HCC cell proliferation. (D) The number of migrated and invaded HCC cells was decreased by TMEM220 overexpression. $* P<0.05$.

\section{TMEM220 mediates the suppressive role of TMEM220-AS1 in HCC cells}

To investigate the regulatory role of TMEM220 in cell proliferation and mobility, we increased TMEM220 levels in MHCC97H and HCCLM3 cells $(P<0.05$, Figure 4A). TMEM220 overexpression significantly suppressed HCC cell proliferation, as indicated by the CCK-8 and EdU assays $(P<0.05$, Figure 4B and 4C). Furthermore, ectopic expression of TMEM220 suppressed the migratory and invasive abilities of HCC cells $(P<0.05$, Figure 4D). TMEM220 levels were downregulated in TMEM220-AS1overexpressing HCCLM3 cells $(P<0.05$, Figure 5A). Notably, TMEM220 silencing reversed the inhibitory effect of TMEM220-AS1 in HCCLM cells $(P<0.05$, Figure 5B-D). Thus, TMEM220 is a functional effector of TMEM220-AS1 in HCC cells.

\section{TMEM220 regulates the $W n t / \beta$-catenin pathway}

To explore the potential mechanism involved in the function of TMEM220 in HCC, KEGG enrichment analysis was conducted to explore the potential signaling pathways affected by TMEM220. We found that low TMEM220 expression was correlated with the Wnt signaling pathway (Figure 6A). Additionally, the subcellular distribution of $\beta$-catenin and the levels of its target genes were detected after TMEM220 overexpression in HCCLM3 cells. As expected, TMEM220 overexpression decreased the nuclear accumulation of $\beta$-catenin and reduced the mRNA levels of MYC, Cyclin D1, and Snail1 $(P<0.05$, Figure $6 \mathrm{~B}$ and 6C). BIO, a GSK3 $\beta$ inhibitor, reversed TMEM220-induced Wnt $/ \beta$-catenin pathway inactivation $(P<0.05$, Figure $6 \mathrm{~B}$ and $6 \mathrm{C})$. Moreover, BIO treatment eliminated the inhibitory role of TMEM220 in HCC cell proliferation and mobility $(P<0.05$, Figure 6D-F). Collectively, TMEM220 exerted a tumor-suppressive role in HCC by inactivating the Wnt/ $\beta$-catenin pathway.

\section{Discussion}

In this study, we first verified that TMEM220AS1 is expressed at low levels in HCC cells. Previous studies have implicated epigenetic and transcriptional 
regulation in the dysregulation of lncRNAs in human cancers [15-17]. For example, DNA hypermethylation leads to a loss of LINC00261 expression in lung cancer [18] and N6-methyladenosine (m6A) modification is responsible for the overexpression of lncRNA RP11 in colorectal cancer [19]. Transcription factors such as p53, Myc, and HIFs, activate lncRNA expression [6, 20, 21]. Thus, it is necessary to elucidate the mechanism underlying the downregulation of TMEM220-AS1 in HCC. LncRNAs are recognized as promising novel diagnostic and prognostic biomarkers for HCC [22, 23]. Serum small extracellular vesicle (EV)-derived LINC00853 is significantly elevated and has been identified as a valuable diagnostic biomarker for early HCC [24]. LINC00978 is highly expressed in the serum of patients with HCC compared with patients with hepatitis and cirrhosis as well as healthy controls [25]. The overexpression of lncRNA H19 predicts poor clinical outcomes in patients with HCC [26]. In this study, we revealed that reduced TMEM220-AS1 levels were closely correlated with advanced tumor stage, venous infiltration, tumor size $>5 \mathrm{~cm}$, and poor prognosis of HCC. Therefore, it is worth determining TMEM220-AS1 expression in the serum of HCC patients and analyzing its diagnostic value in future studies.

Functional experiments confirmed that TMEM220-AS1 overexpression inhibited the growth and mobility of HCC cells. As an antisense lncRNA of TMEM220, TMEM220-AS1 positively regulates TMEM220 expression in HCC. A previous study showed that AdipoQ AS lncRNA binds to AdipoQ mRNA and suppresses its translation [27]. The lncRNA FOXC2-AS1 transcriptionally and posttranscriptionally enhances FOXC2 expression in osteosarcoma [28]. TMEM220-AS1 overexpression consistently increased TMEM220 mRNA and protein levels in HCC cells. TMEM220 mRNA stability was not affected by TMEM220-AS1 overexpression in HCC cells. These results suggest that TMEM220-AS1 transcriptionally regulates TMEM220 expression in HCC cells.

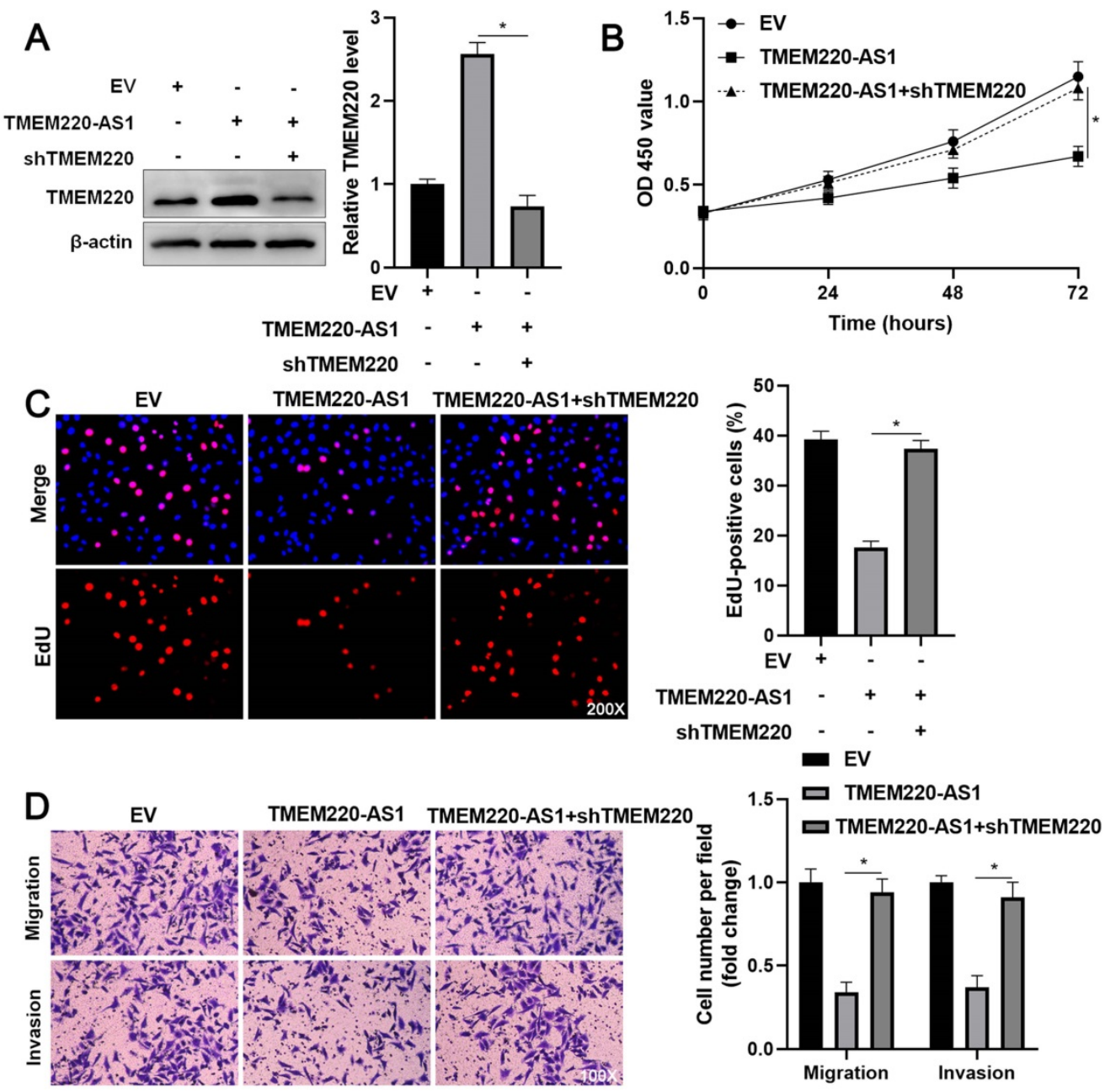

Figure 5. TMEM220 knockdown reverses the inhibitory effect of TMEM220-AS1 in HCCLM3 cells. (A) Western blot analysis showed that TMEM220 levels were reduced by TMEM220 shRNA (shTMEM220) in TMEM220-AS1-overexpressing HCCLM3 cells. (B) MTT, (C) EdU, and (D) transwell analyses demonstrated that TMEM220 knockdown reversed the suppressive role of TMEM220-AS1 in HCCLM3 cells. ${ }^{*} P<0.05$. 

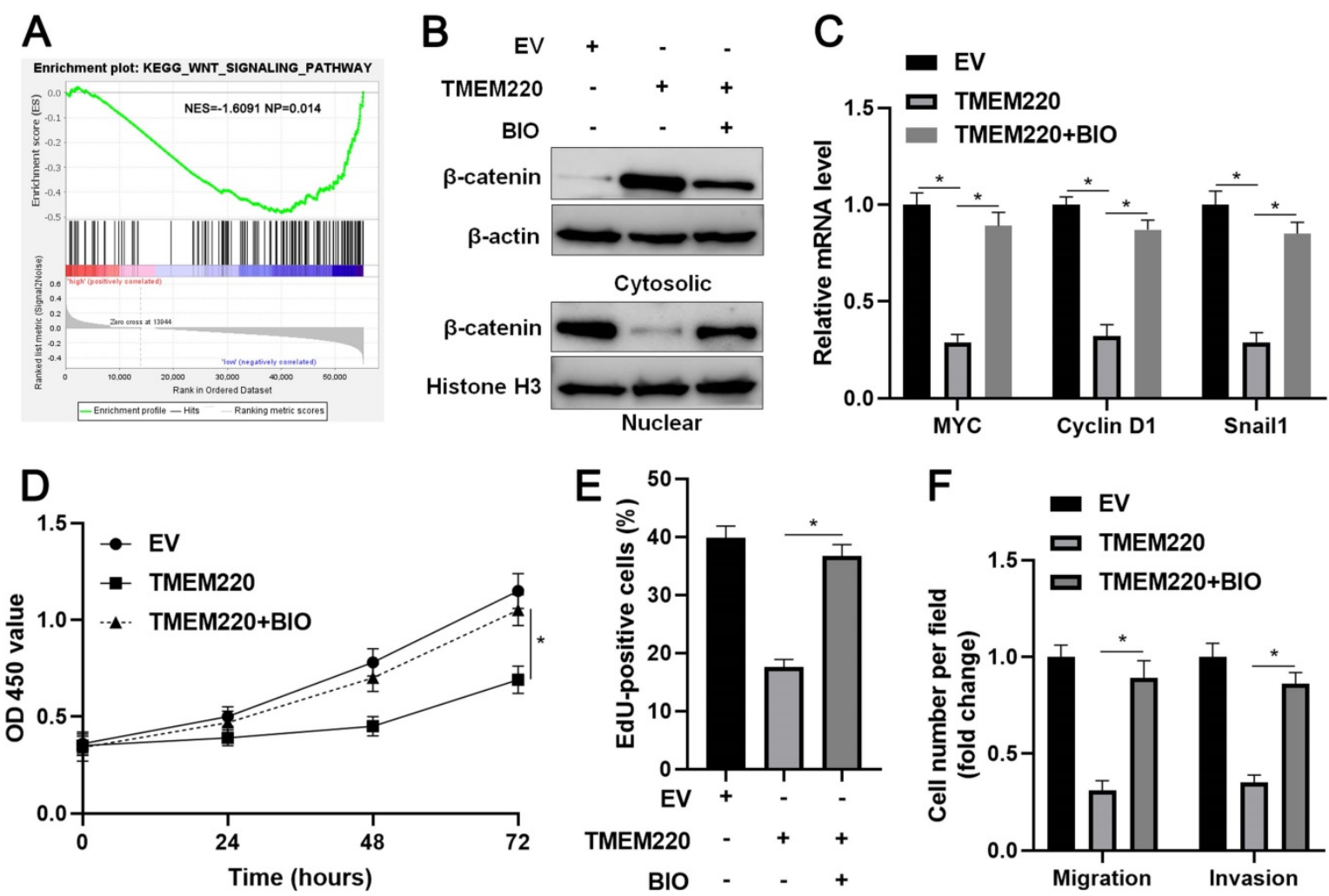

Figure 6. TMEM220 regulates the Wnt/ $\beta$-catenin pathway in HCC cells. (A) KEGG enrichment analysis showed that the low TMEM220 level was correlated with the Wnt signaling pathway. (B) TMEM220-overexpressing HCCLM3 cells were treated with $2 \mu M$ of the GSK3 inhibitor, BIO, and western blotting was performed to detect $\beta$-catenin. (C) TMEM220-overexpressing HCCLM3 cells were treated with $2 \mu M$ of the GSK3 $\beta$ inhibitor, BIO, and RT-qPCR was performed to detect MYC, Cyclin D1, and Snaill mRNA levels. (D) MTT, (E) EdU, and (F) transwell analyses demonstrated that BIO treatment reversed the suppressive role of TMEM220 in HCCLM3 cells. *P<0.05.

TMEM205 and TMEM106C, two members of the TMEM family, are highly expressed in HCC [29, 30]. TMEM205 is recognized as an independent prognostic marker and is correlated with the immune microenvironment in HCC [30]. The upregulation of TMEM106C predicts poor HCC prognosis and enhances the malignancy of cancer cells [29]. Conversely, our data showed that TMEM220 is downregulated in HCC. The reduced level of TMEM220 indicated reduced survival in patients with HCC. TMEM220 overexpression suppressed HCC cell migration, invasion, and proliferation, which was similar to the effects of TMEM220-AS1 on HCC cells. TMEM220 knockdown eliminated the role of TMEM220-AS1 in HCC cells. Collectively, TMEM220 is a tumor suppressor that mediates the anti-HCC effects of TEMEM220-AS1. KEGG enrichment analysis indicated that TMEM220 is inversely associated with the Wnt signaling pathway. TMEM220 overexpression significantly reduced the nuclear translocation of $\beta$-catenin and decreased the levels of MYC, cyclin D1, and Snail1 mRNA in HCC cells. The GSK3 $\beta$ inhibitor, BIO, a Wnt $/ \beta$-catenin agonist, eliminated TMEM220-induced Wnt/ $\beta$-catenin pathway inactivation. Several studies have demonstrated that the $\mathrm{Wnt} / \beta$-catenin pathway plays a role in the occurrence and progression of HCC by enhancing target gene expression, including MYC, cyclin D1, and Snail1 [31-33]. We found that BIO treatment also enhanced the migration, invasion, and proliferation of TMEM220-overexpressing HCCLM3 cells. Thus, TMEM220 exerted a tumor-suppressive role in HCC by inactivating the Wnt/ $\beta$-catenin pathway.

In summary, we demonstrated the downregulation of TMEM220-AS1 and TMEM220 in HCC and verified their potential in predicting poor prognosis. Both TMEM220-AS1 and TMEM220 inhibited the migration, invasion, and proliferation of HCC cells. TMEM220 mediated TMEM220-AS1's anti-HCC effects by inactivating the $W n t / \beta$-catenin pathway. TMEM220-AS1 and TMEM220 may be promising prognostic biomarkers and therapeutic targets for HCC.

\section{Abbreviations}

IARC: International Agency for Research on Cancer; HCC: hepatocellular carcinoma; lncRNA: long noncoding RNA; TMEM220-AS1: transmembrane protein 220 antisense RNA 1; TCGA: The Cancer Genome Atlas; m6A: N6-methyladenosine; EV: extracellular vesicle. 


\section{Supplementary Material}

Supplementary figures and table.

http://www.jcancer.org/v12p6805s1.pdf

\section{Acknowledgements}

This work was supported by grants from the Zhejiang Provincial Natural Science Foundation of China (LY19H160036), the Key Research and Development Program of Shaanxi (2020SF-068), the Key Research and Development Provincial Program (2020C03008), and the Co-construction of Provincial and Department Project (WKJ-ZJ-1919).

\section{Competing Interests}

The authors have declared that no competing interest exists.

\section{References}

1. Sung H, Ferlay J, Siegel RL, Laversanne M, Soerjomataram I, Jemal A, et al. Global Cancer Statistics 2020: GLOBOCAN Estimates of Incidence and Mortality Worldwide for 36 Cancers in 185 Countries. CA Cancer J Clin. 2021; 71: 209-49.

2. Zhang B, Zhang Z, Huang Z, Chen Y, Chen M, Bie P, et al. 42,573 cases of hepatectomy in China: a multicenter retrospective investigation. Sci China Life Sci. 2018; 61: 660-70.

3. Schmitt AM, Chang HY. Long Noncoding RNAs in Cancer Pathways. Cancer Cell. 2016; 29: 452-63.

4. Lim LJ, Wong SYS, Huang F, Lim S, Chong SS, Ooi LL, et al. Roles and Regulation of Long Noncoding RNAs in Hepatocellular Carcinoma. Cancer Res. 2019; 79: 5131-9.

5. Wang Y, Liu Z, Yao B, Li Q, Wang L, Wang C, et al. Long non-coding RNA CASC2 suppresses epithelial-mesenchymal transition of hepatocellular carcinoma cells through CASC2/miR-367/FBXW7 axis. Mol Cancer. 2017; 16: 123.

6. Wang L, Sun L, Liu R, Mo H, Niu Y, Chen T, et al. Long non-coding RNA MAPKAPK5-AS1/PLAGL2/HIF-1alpha signaling loop promotes hepatocellular carcinoma progression. J Exp Clin Cancer Res. 2021; 40: 72.

7. Wang Y, Yang L, Chen T, Liu X, Guo Y, Zhu O, et al. A novel IncRNA MCM3AP-AS1 promotes the growth of hepatocellular carcinoma by targeting miR-194-5p/FOXA1 axis. Mol Cancer. 2019; 18: 28.

8. Liu Z, Mo H, Sun L, Wang L, Chen T, Yao B, et al. Long noncoding RNA PICSAR/miR-588/EIF6 axis regulates tumorigenesis of hepatocellular carcinoma by activating PI3K/AKT/mTOR signaling pathway. Cancer Sci. 2020; 111: 4118-28.

9. Xiao Z, Liu Y, Zhao J, Li L, Hu L, Lu Q, et al. Long noncoding RNA LINC01123 promotes the proliferation and invasion of hepatocellular carcinoma cells by modulating the miR-34a-5p/TUFT1 axis. Int J Biol Sci. 2020; 16: 2296-305.

10. Bai J, Yao B, Wang $L$, Sun $L$, Chen $T$, Liu $R$, et al. IncRNA A1BG-AS1 suppresses proliferation and invasion of hepatocellular carcinoma cells by targeting miR-216a-5p. J Cell Biochem. 2019; 120: 10310-22.

11. Du W, Chen W, Shu Z, Xiang D, Bi K, Lu Y, et al. Identification of prognostic biomarkers of hepatocellular carcinoma via long noncoding RNA expression and copy number alterations. Epigenomics. 2020; 12: 1303-15.

12. Choi B, Han TS, Min J, Hur K, Lee SM, Lee HJ, et al. MAL and TMEM220 are novel DNA methylation markers in human gastric cancer. Biomarkers. 2017; 22: $35-44$

13. Dou C, Sun L, Wang L, Cheng J, Wu W, Zhang C, et al. Bromodomaincontaining protein 9 promotes the growth and metastasis of human hepatocellular carcinoma by activating the TUFT1/AKT pathway. Cell Death Dis. 2020; 11: 730 .

14. Tang Z, Li C, Kang B, Gao G, Zhang Z. GEPIA: a web server for cancer and normal gene expression profiling and interactive analyses. Nucleic Acids Res. 2017; 45: W98-W102

15. Zhao X, Chen Q, Cai Y, Chen D, Bei M, Dong H, et al. TRA2A Binds With LncRNA MALAT1 To Promote Esophageal Cancer Progression By Regulating EZH2/beta-catenin Pathway. J Cancer. 2021; 12: 4883-90.

16. Ren R, Du Y, Niu X, Zang R. ZFPM2-AS1 transcriptionally mediated by STAT1 regulates thyroid cancer cell growth, migration and invasion via miR-515-5p/TUSC3. J Cancer. 2021; 12: 3393-406.

17. Coker H, Wei G, Brockdorff N. m6A modification of non-coding RNA and the control of mammalian gene expression. Biochim Biophys Acta Gene Regul Mech. 2019; 1862: 310-8.
18. Shahabi S, Kumaran V, Castillo J, Cong Z, Nandagopal G, Mullen DJ, et al. LINC00261 Is an Epigenetically Regulated Tumor Suppressor Essential for Activation of the DNA Damage Response. Cancer Res. 2019; 79: 3050-62.

19. Wu Y, Yang X, Chen Z, Tian L, Jiang G, Chen F, et al. m(6)A-induced lncRNA RP11 triggers the dissemination of colorectal cancer cells via upregulation of Zeb1. Mol Cancer. 2019; 18: 87.

20. Olivero CE, Martinez-Terroba E, Zimmer J, Liao C, Tesfaye E, Hooshdaran N, et al. p53 Activates the Long Noncoding RNA Pvt1b to Inhibit Myc and Suppress Tumorigenesis. Mol Cell. 2020; 77: 761-74 e8.

21. Hua O, Jin M, Mi B, Xu F, Li T, Zhao L, et al. LINC01123, a c-Myc-activated long non-coding RNA, promotes proliferation and aerobic glycolysis of non-small cell lung cancer through miR-199a-5p/c-Myc axis. J Hematol Oncol. 2019; $12: 91$.

22. Sasaki R, Kanda T, Yokosuka O, Kato N, Matsuoka S, Moriyama M. Exosomes and Hepatocellular Carcinoma: From Bench to Bedside. Int J Mol Sci. 2019; 20: 1406.

23. Huang Z, Zhou JK, Peng Y, He W, Huang C. The role of long noncoding RNAs in hepatocellular carcinoma. Mol Cancer. 2020; 19: 77.

24. Kim SS, Baek GO, Ahn HR, Sung S, Seo CW, Cho HJ, et al. Serum small extracellular vesicle-derived LINC00853 as a novel diagnostic marker for early hepatocellular carcinoma. Mol Oncol. 2020; 14: 2646-59.

25. Xu X, Gu J, Ding X, Ge G, Zang X, Ji R, et al. LINC00978 promotes the progression of hepatocellular carcinoma by regulating EZH2-mediated silencing of p21 and E-cadherin expression. Cell Death Dis. 2019; 10: 752

26. Ye Y, Guo J, Xiao P, Ning J, Zhang R, Liu P, et al. Macrophages-induced long noncoding RNA H19 up-regulation triggers and activates the miR-193b/MAPK1 axis and promotes cell aggressiveness in hepatocellular carcinoma. Cancer Lett. 2020; 469: 310-22.

27. Cai R, Sun Y, Qimuge N, Wang G, Wang Y, Chu G, et al. Adiponectin AS lncRNA inhibits adipogenesis by transferring from nucleus to cytoplasm and attenuating Adiponectin mRNA translation. Biochim Biophys Acta Mol Cell Biol Lipids. 2018; 1863: 420-32.

28. Zhang $\mathrm{CL}$, Zhu $\mathrm{KP}, \mathrm{Ma} \mathrm{XL}$. Antisense lncRNA FOXC2-AS1 promotes doxorubicin resistance in osteosarcoma by increasing the expression of FOXC2. Cancer Lett. 2017; 396: 66-75.

29. Duan J, Qian Y, Fu X, Chen M, Liu K, Liu H, et al. TMEM106C contributes to the malignant characteristics and poor prognosis of hepatocellular carcinoma. Aging (Albany NY). 2021; 13: 5585-606.

30. Rao J, Wu X, Zhou X, Deng R, Ma Y. TMEM205 Is an Independent Prognostic Factor and Is Associated With Immune Cell Infiltrates in Hepatocellular Carcinoma. Front Genet. 2020; 11: 575776.

31. Perugorria MJ, Olaizola P, Labiano I, Esparza-Baquer A, Marzioni M, Marin JJG, et al. Wnt-beta-catenin signalling in liver development, health and disease. Nat Rev Gastroenterol Hepatol. 2019; 16: 121-36.

32. Huang J, Qu Q, Guo Y, Xiang Y, Feng D. Tankyrases/beta-catenin Signaling Pathway as an Anti-proliferation and Anti-metastatic Target in Hepatocarcinoma Cell Lines. J Cancer. 2020; 11: 432-40.

33. Wang $Y$, Sun L, Wang L, Liu Z, Li Q, Yao B, et al. Long non-coding RNA DSCR8 acts as a molecular sponge for miR-485-5p to activate Wnt/betacatenin signal pathway in hepatocellular carcinoma. Cell Death Dis. 2018; 9: 851. 